\title{
Noise predictions for STM in systems with local electron nematic order
}

\author{
Y. L. Loh, ${ }^{1,2}$ E. W. Carlson, ${ }^{2}$ and K. A. Dahmen ${ }^{3}$ \\ ${ }^{1}$ Department of Physics, The Ohio State University, Columbus, Ohio 43210, USA \\ ${ }^{2}$ Department of Physics, Purdue University, West Lafayette, Indiana 47907, USA \\ ${ }^{3}$ Department of Physics, University of Illinois, Urbana-Champaign, Illinois 61801, USA
}

(Received 19 November 2009; revised manuscript received 11 May 2010; published 17 June 2010)

\begin{abstract}
We propose that thermal noise in local stripe orientation should be readily detectable via STM on systems in which local stripe orientations are strongly affected by quenched disorder. Stripes, a unidirectional, nanoscale modulation of electronic charge, are strongly affected by quenched disorder in two-dimensional and quasitwo-dimensional systems. While stripe orientations tend to lock to major lattice directions, dopant disorder locally breaks rotational symmetry. In a host crystal with otherwise $C_{4}$ rotational symmetry, stripe orientations in the presence of quenched disorder map to the random field Ising model. While the low-temperature state of such a system is generally a stripe glass in two dimensional or strongly layered systems, as the temperature is raised, stripe orientational fluctuations become more prevalent. We propose that these thermally excited fluctuations should be readily detectable in scanning tunneling spectroscopy as telegraph noise in the high-voltage part of the local $I(V)$ curves. We predict the spatial, temporal, and thermal evolution of such noise, including the circumstances under which such noise is most likely to be observed. In addition, we propose an in situ test, amenable to any local scanning probe, for assessing whether such noise is due to correlated fluctuations rather than independent switchers.
\end{abstract}

DOI: 10.1103/PhysRevB.81.224207

PACS number(s): 74.50. $+\mathrm{r}, 72.70 .+\mathrm{m}, 75.10 . \mathrm{Nr}$

\section{INTRODUCTION}

There is experimental evidence that many strongly correlated electronic systems such as nickelates, cuprates, and manganites exhibit some degree of local inhomogeneity, i.e., nanoscale variations in the local electronic properties. Describing the electronic behavior of these materials involves several degrees of freedom, including orbital, spin, charge, and lattice degrees of freedom. Disorder only compounds the problem. Not only can certain types of disorder destroy phase transitions, leaving mere crossovers in their wake, it can fundamentally alter ground states, sometimes forbidding long range order. Especially in systems where different physical tendencies compete, disorder can act as nucleation points for competing ground states. The interplay between many degrees of freedom, strong correlations, and disorder can lead to a hierarchy of length scales over which the resulting physics must be described. While such electronic systems are highly susceptible to pattern formation at the nanoscale, unfortunately most of our theoretical and experimental tools are designed for understanding and detecting homogeneous phases of matter.

Because of these difficulties, the presence of quenched disorder in real materials can make it exceedingly difficult to discern the character of the local pattern formation. One might expect to overcome the difficulty by growing cleaner samples, but certain forms of local order (such as stripe orientations, i.e., the nematic component of stripes) are so fragile that any finite disorder precludes a long range ordered ground state in two dimensions. (Finite but small disorder can preclude it in layered systems.) It is therefore desirable to develop theoretical and experimental techniques aimed at characterizing and detecting local electronic order. In previous work, we have shown that noise and nonequilibrium effects can be used to help illuminate the local electronic tendencies. ${ }^{1}$ In this paper, we discuss the implications of such noise for scanning tunneling microscopy. In particular, we propose that thermally excited switching of local stripe orientations should be readily detectable via scanning noise microscopy, appearing as telegraph noise in the high-voltage part of atomic scale $d I / d V$ curves.

Long-range stripe order has been detected in a subset of cuprate oxides, where it can coexist with superconductivity, as well as in the related nickel oxides. ${ }^{2}$ However, most cuprate superconductors lack evidence of long-range stripe order. This calls into question theories of cuprate superconductivity based on quasi-one-dimensional electronic structure. On the other hand, because the pairing scale is large, it can in principle be established on short length scales. Scanning tunneling microscopy (STM) experiments have shown that the correlation length of the superconducting order parameter, i.e., the distance over which the gap scale can vary, is about 10-20 ̊.) Thus the salient question is not whether longrange stripe order is ubiquitous in cuprate superconductors, but whether short-range stripe order is ubiquitous. Nevertheless, the fact that long-range ordered stripes have been observed in several materials with strong electronic correlations means that the study of these structures in the presence of quenched disorder is interesting in its own right, regardless of issues of the mechanism of cuprate superconductivity.

Indeed, recent STM experiments show evidence of an electronic cluster glass of locally unidirectional domains in $\mathrm{Na}-\mathrm{CCOC}$ and Dy-Bi2212 at low temperature. ${ }^{3}$ We predict that upon raising the temperature in such systems, more and more stripe domains will become thermally excited, resulting in local fluctuations in the stripe orientation. The main purpose of this paper is to predict the spatial, temporal, and thermal evolution of such noise. The effect of stripes on STM tunneling spectra and their spatial Fourier transforms is reviewed in Ref. 4. 
Telegraph noise in the mesoscopic transport properties of a YBCO nanowire has been reported by Bonetti et al. in the pseudogap regime, ${ }^{5}$ appearing on time scales of about $10-50$ $\mathrm{s}$, and producing resistance fluctuations of the order of $0.5 \%$ of the total resistance of the wire. We have previously modeled this telegraph noise via a mapping of stripes in the presence of quenched disorder to the random-field Ising model ${ }^{1}$ (RFIM) (as explained below), along with a further mapping to a related random resistor network. While the nanowire transport results can be captured by this model, it was not possible to definitively rule out other sources of the telegraph noise, such as superconducting fluctuations. ${ }^{5}$ Transport noise has also been reported on time scales of $1-1000 \mathrm{~s}$ in the $c$-axis resistivity of bulk LSCO at $T \leqq 0.3 \mathrm{~K}$ by Raičević and co-workers. ${ }^{6}$ In addition, an increase in the resistance noise has been reported in BSSCO in the pseudogap regime. ${ }^{7}$

Is the low-temperature glass of unidirectional domains observed in Na-CCOC and Dy-Bi2212 related to the transport noise observed in YBCO, LSCO, and BSCCO? If similar noise were to be found with STM, it would begin to bridge the gap between the real-space imaging capability of that probe and the noise seen in mesoscopic and macroscopic systems. This would allow concrete connections to be made between the microscopic and macroscopic behavior and provide guidance as to how to extract information about local order from macroscopic probes when microscopic probes such as STM are not feasible. Furthermore, since transport noise is a bulk measurement, finding evidence of similar noise in STM would establish a connection between physics happening in the bulk and that occurring on the surface. In addition, the wealth of information available from STM can be used to set the parameters of our model so that concrete predictions can then be made for other measurements.

We now describe the mapping of stripe orientations in the presence of quenched disorder to the random-field Ising model. ${ }^{1}$ Stripes in a host crystal such as the cuprates and nickelates tend to lock to favorable lattice directions, often either vertically or horizontally along $\mathrm{Cu}-\mathrm{O}(\mathrm{Ni}-\mathrm{O})$ bond directions. This breaks the symmetry of the host crystal from fourfold to twofold rotational symmetry. With two allowed orientations, the nematic component of the stripe order parameter is of Ising character, where the Ising variable or "pseudospin" $\sigma= \pm 1$ corresponds to horizontal or vertical stripe patches. The tendency of neighboring stripe patches to align is modeled as a ferromagnetic interaction between nearest-neighbor Ising variables. Dopant atoms act like a random field on the electronic stripe nematic so that in any given region, the particular dopant arrangement will favor one or the other orientation of the stripes locally, producing a disorder-dependent pattern of stripe patches with two orientations. Thus stripe orientations in a host crystal with dopant disorder ${ }^{8,9}$ can be mapped to the RFIM, ${ }^{1}$

$$
H=-J \sum_{\langle i j\rangle_{\|}} \sigma_{i} \sigma_{j}-J_{\perp} \sum_{\langle i j\rangle_{\perp}} \sigma_{i} \sigma_{j}-\sum_{i}\left(h+h_{i}\right) \sigma_{i},
$$

where $J$ is the in-plane coupling, $J_{\perp}$ is the interlayer coupling, $h$ is an orienting field, and $h_{i}$ are the random pseudofields at each site. In the summations, $\langle i j\rangle_{\|}$denotes a summation over sites within a plane, and $\langle i j\rangle_{\perp}$ denotes
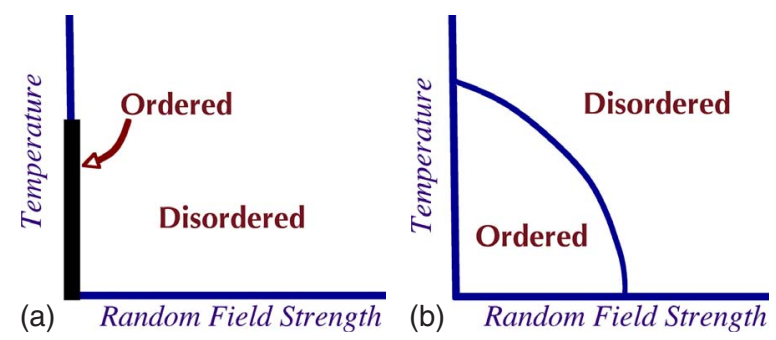

FIG. 1. (Color online) Schematic phase diagram of the random field Ising model. (a) In two dimensions, the critical disorder strength is zero, and for any finite disorder strength the model is in a disordered phase at all temperatures. (b) In three dimensions, there is a finite critical disorder strength.

summation over sites in neighboring planes. The orienting field may be, for example, $h \propto B_{x}^{2}-B_{y}^{2}$ where $\vec{B}$ is the physical external magnetic field. ${ }^{10}$ The random fields $h_{i}$ are chosen independently from a Gaussian distribution with standard deviation $R$ (the disorder strength). The order parameter $M$ $=\frac{1}{N} \Sigma_{i}\left\langle\sigma_{i}\right\rangle$ represents the degree of stripe orientation, i.e., nematic order. The size of a stripe patch sets the spacing of the Ising lattice, and we may use information from experiments to set bounds on this lattice spacing. For example, for the purposes of comparing with STM experiments on NaCCOC and Dy-Bi2212, ${ }^{3}$ a reasonable lattice spacing would be $16 \AA$.

One might imagine that two vertical stripe patches which are nearest neighbors along the $x$ direction have different interaction energy from two vertical stripe patches which are nearest neighbors along the $y$ direction. Indeed, this type of compass term for the Ising nematic with short-range interactions can be constructed as well. The addition of such a term reduces to an effective internal field, which formally sums to zero in the bulk. However, it can be nonzero along boundaries of the sample, indicating a tendency for edges of the sample to align stripes. Since we are interested in the physics of the bulk, we have not included such effects, although it should be important in confined geometries. Local strain fields associated with coupling between the electron nematic and the lattice may produce similar energy differences, but with longer ranges interactions and martensitic physics, ${ }^{11}$ that limit the domain size. Nevertheless, the main features of the model-glassy behavior and correlated dynamics over long time scales-remain intact.

The materials of interest are layered materials, corresponding to a quasi-two-dimensional system. As shown schematically in Fig. 1, for any finite disorder strength, the two-dimensional (2D) RFIM remains disordered at all temperatures in the thermodynamic limit. ${ }^{12}$ In three dimensions, the RFIM can order below a critical disorder strength. For the finite layered systems we consider here, there is a finite critical disorder strength, although it may be quite suppressed for strongly layered systems. This implies that strongly layered materials are less likely to support an ordered Ising nematic phase than materials with stronger coupling in the $c$ direction. Although the charge component of stripes is subject to Coulomb coupling from plane to plane, because they are overall charge neutral this coupling is greatly diminished for distances longer than the local modu- 


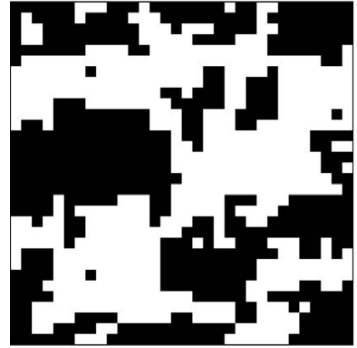

(a)Color visualization

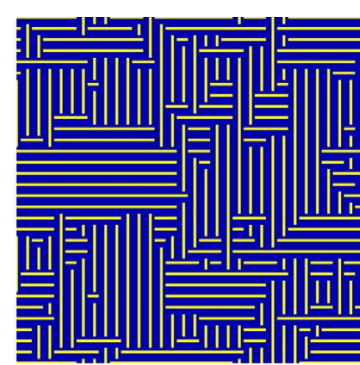

(b)Stripe visualization
FIG. 2. (Color online) A snapshot of a layered random-field Ising model with $R=2.5 \mathrm{~J}$ and $T=0.5 \mathrm{~J}$ on a $32 \times 32 \times 8$ cubic lattice (see text for details). Only the top layer is shown. (a) Here, the two states of the Ising variables, $\sigma_{i}= \pm 1$, are represented by white and black patches. (b) The same results visualized as vertical and horizontal stripes.

lation wavelength (about $16 \AA$ ), and the short-range interactions of our model are sufficient to describe the $c$-axis coupling.

\section{THERMALLY ACTIVATED STRIPE ORIENTATION SWITCHING}

In Fig. 2, we show an example of the low-temperature behavior in the disordered phase, revealing a lowtemperature pattern with locally unidirectional domains of various sizes. Figure 2(a) shows a single thermalized configuration in our model, and Fig. 2(b) shows the corresponding stripe visualization. In order to do the calculation, we have performed Monte Carlo simulations of Eq. (1) with checkerboard Glauber updates $^{13}$ for a layered RFIM on a $32 \times 32 \times 8$ cubic lattice. We use periodic boundary conditions in the $x$ and $y$ directions along with open boundary conditions in the $z$ direction, and we show results from the top layer, as a rough approximation to the geometry of an STM experiment on a surface. We have used coupling constants appropriate to a layered system, $J=1$ within each layer and $J_{z}=0.125$ between layers, with a disorder strength $R$ $=2.5 \mathrm{~J}$, which is above the critical disorder strength. This typical configuration is taken after approximately $10^{7}$ timesteps. (It takes about $5 \times 10^{4}$ steps to thermalize at these parameters.) In Fig. 2(a), we show a conventional visualization of the Ising state, using color to represent the state of the spin. In Fig. 2(b), we have shown the director of the Ising nematic, in order to represent the average direction in which stripes run at any given site in the corresponding Ising model.

Because we have used a disorder strength which is larger than the critical disorder strength, the ground-state configuration is dominated by quenched disorder, and although there are domains of various sizes, the average domain size is small. A consequence of this is that upon thermal cycling, the low-temperature state returns to the same pattern of stripe orientations, since the local orientation of each stripe patch is dominated by the quenched disorder rather than by the thermal history. The tunneling asymmetry maps of Ref. 3 bear a strong resemblance to configurations of the RFIM [see Fig. 2(b)]. Note that in Fig. 2 we have shown a single (typical) configuration, rather than an ensemble average. We believe that this is the correct comparison for STM experiments at $T=4.2 \mathrm{~K}$ (as opposed to an ensemble average), since the time scales over which a single $d I / d V$ curve is recorded are small compared to the switching time of stripes at that temperature. $^{14}$

While most fluctuations are frozen out at low temperature, upon raising the temperature, certain regions of the system become active and exhibit thermally driven stripe orientation fluctuations. In an STM experiment, this should lead to local telegraph noise in the active regions, where some atomic positions will alternate between having a charge stripe on them or not. We show below where to look for such noise as a function of position and temperature and then describe an algorithm for detecting whether such noise is due to correlation effects rather than from some local noninteracting source.

Note that microscopically, a rigid rotation is not required in order to produce the thermally fluctuating local Ising variable of our model. Rather, minor local rearrangements are sufficient to change the direction of any particular unidirectional domain. Exactly how such a local rearrangement transpires is a question of the local energy barriers of a disordered many-body system and will be dominated by whatever is the lowest local activation energy in any given region. Our proposal is independent of these microscopic details. It is, of course, conceivable that other mechanisms (e.g., side-to-side stripe fluctuations in a disordered system or fluctuations of other inhomogeneous patterns) may give rise to fluctuating Ising variables.

Because the materials in question are extremely complicated and each Ising variable in our model encompasses many electrons, it is difficult to arrive at a reliable microscopic estimate of time scales. Nevertheless, here is a rough argument to show that the numbers are not unreasonable. Assuming that the stripe fluctuations involve electron dynamics, the attempt frequency would be of the order of the Fermi velocity $(2 \mathrm{eV} \AA)$ divided by the stripe spacing (16 $\AA$ ), that is, $10^{15} \mathrm{~Hz}$. Noise in the YBCO nanowire was observed on a time scale of about $50 \mathrm{~s}$ at $100 \mathrm{~K} .^{5}$ For an Arrhenius type law, this corresponds to a local energy barrier of about $3800 \mathrm{~K}$, not far from the bare Heisenberg spin exchange couplings in the cuprates. While the Heisenberg coupling is not equal to our Ising coupling, it is one parameter, which sets the Ising coupling, and so the two may be of the same magnitude. Note, however, that due to the randomness in our model, every cluster has a different time scale, many clusters have multiple time scales, and these time scales span several orders of magnitude. As a result, the issue is less one of whether time scales will be accessible experimentally, and more one of where spatially to look for fluctuating domains. We address this issue in Fig. 3.

Figure 3 shows what happens when the temperature is raised on the same system as that in Fig. 2. In the leftmost column of Fig. 3, the purple regions are exhibiting stripe orientation fluctuations. In the corresponding stripe visualization (second column of Fig. 3), this appears as places where the different stripe orientations appear to "invade" 


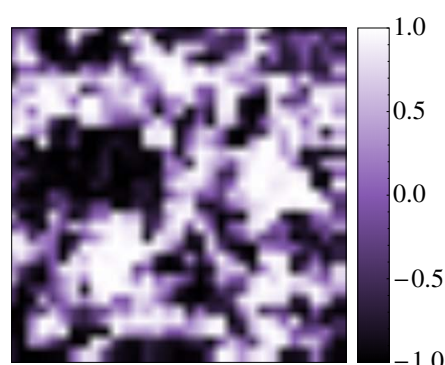

(a)

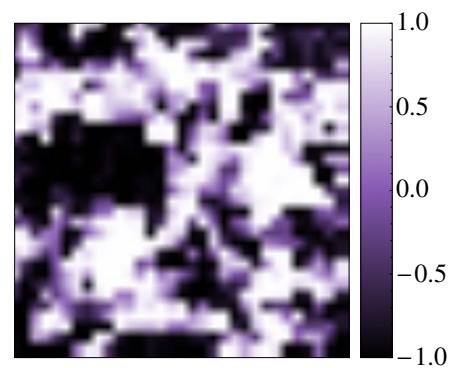

(e)

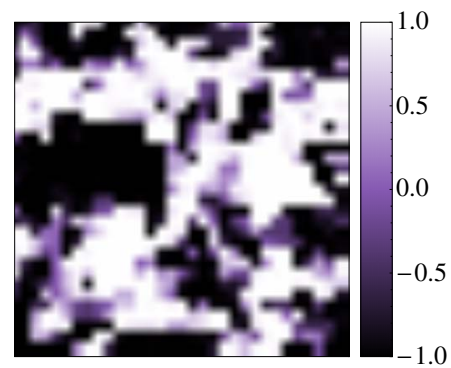

(i)

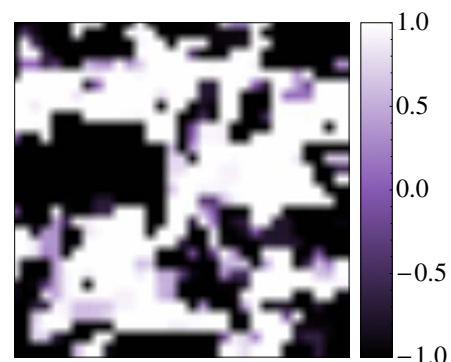

(m)

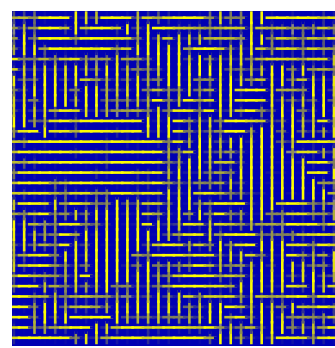

(b)

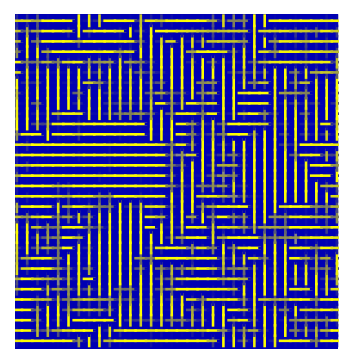

(f)

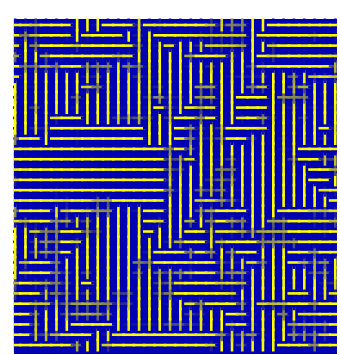

(j)

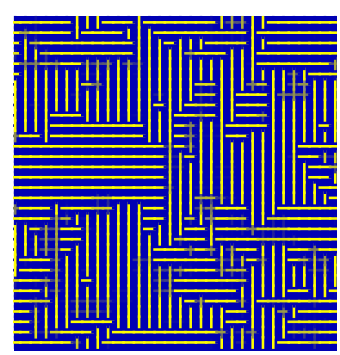

(n)

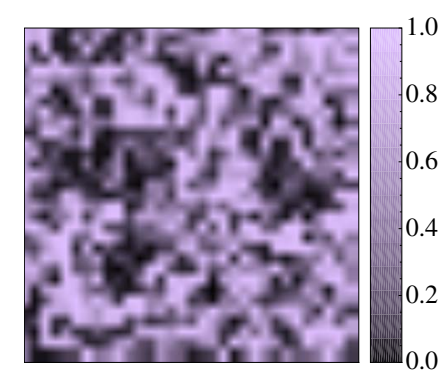

(c)

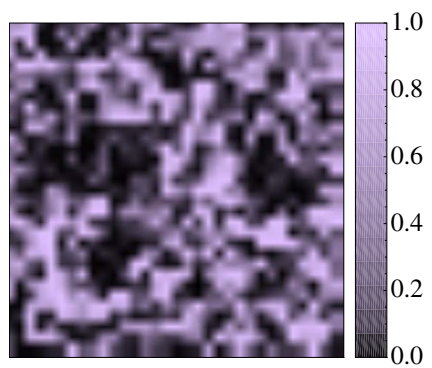

(g)

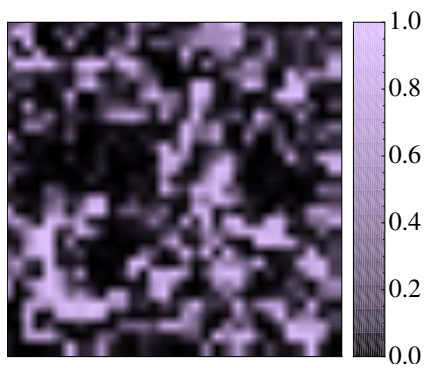

(k)

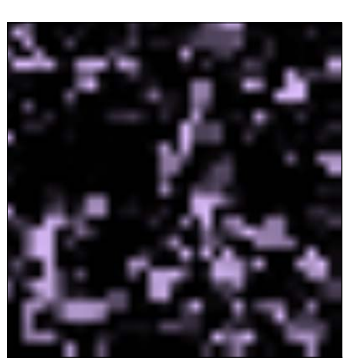

(o)

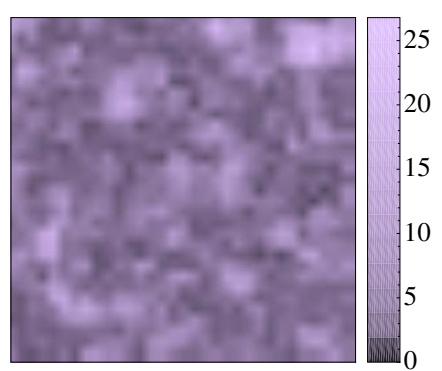

(d)

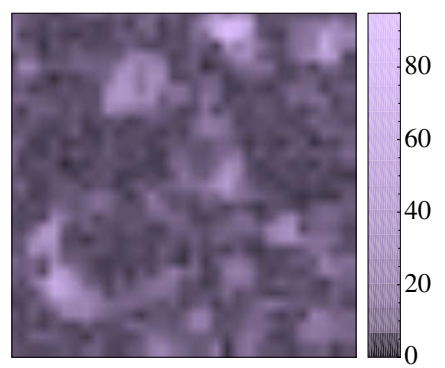

(h)

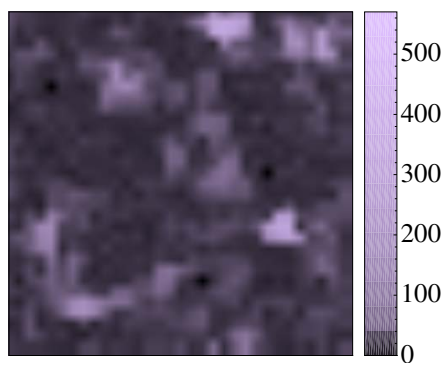

(I)

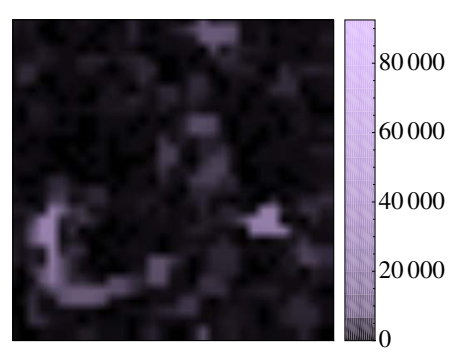

(p)

FIG. 3. (Color online) Fluctuations of the RFIM for a disorder strength of $R=2.5 \mathrm{~J}$ at temperatures $T / \mathrm{J}=2,1.5,1.0 ., 0.5$ (top to bottom) on a $32 \times 32 \times 8$ cubic lattice (see text for details). Only the top layer is shown. In the leftmost column, we show the time-averaged (pseudo)spins $\left\langle\sigma_{i}\right\rangle$, where white and black denote positive and negative averages, respectively, with purple denoting average pseudospins near zero. In the next column, we plot each averaged pseudospin variable as the director of the Ising nematic, to visualize the pattern of horizontal and vertical stripes. Black sites from the first column correspond to horizontal stripe orientation, and white sites from the first column correspond to vertical stripe orientation. The third column shows the variance of the (pseudo)spins, $\left\langle\left(\Delta \sigma_{i}\right)^{2}\right\rangle$. The rightmost column shows the local switching time scale $\tau_{i}$ (see text for definition). At low temperatures, fluctuations are all but frozen out, and local switching time scales diverge. As the temperature is raised, more and more clusters become active, showing as bright spots in the variance maps (third column). Note that the boundaries of the low temperature clusters become active first as temperature is raised. At high temperature, noise is apparent throughout the sample.

each other. These active regions have relatively high variance compared to the surrounding regions, as shown in the third column of Fig. 3. The purple patches in the leftmost column can be seen as active (light) patches in the variance maps. As temperature is raised, more and more regions in the sample become "active." The places which become active first are boundaries between the low temperature (frozen) domains, and especially domain boundaries with several small domains. These are the best places to look for local telegraph noise upon raising temperature. 

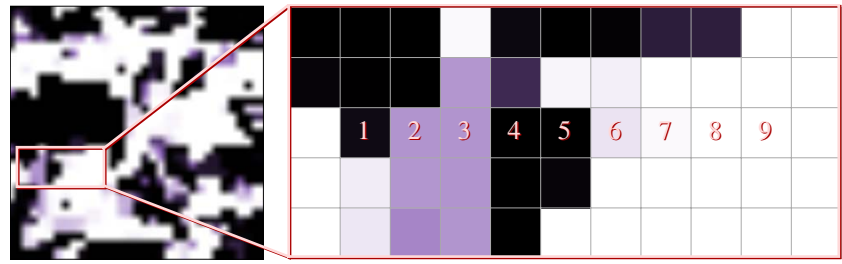

FIG. 4. (Color online) A line of sites in the RFIM at $R=2.5 \mathrm{~J}$ and $T=0.5 \mathrm{~J}$. This corresponds to the simulation from the fourth row of Fig. 3, and we have chosen a line of sites going through an "active" region. The numbered sites correspond to the time traces in Fig. 5 and the block variances in Fig. 8 .

Of course, the observation of telegraph noise is not sufficient to determine whether correlations are present in the system. Telegraph noise could arise from other sources, such as an unstable defect on the tip, or even defect hopping on the surface of the material itself. However, these are uncorrelated sources of noise due to isolated, independent switchers. We are interested in how to identify noise arising from a correlated system. One way to distinguish such "spurious" possible sources of telegraph noise from that due to correlations in an interacting model such as the one considered here is to analyze the power spectrum of a time series of the data. It is well known that for a "stationary" random telegraph signal (i.e., one with a constant probability of flipping from "up" to "down," and with a separate unchanging probability of flipping from "down" to "up") the resulting power spectrum is a Lorentzian, ${ }^{15,16}$ and deviations from this form would presumably indicate correlations. ${ }^{17}$ However in the materials of interest, where the time scales have been observed to be as long as 10-50 s in YBCO (Ref. 5) and 1000 $s$ in $\mathrm{LBCO},{ }^{6}$ a time domain criterion is much more desirable.

We first describe what we mean by correlated switchers, and then we present a criterion for detecting spatial correlations using a time domain analysis at a single site. In Fig. 4, we show a few sites in a larger system. We have chosen some sites that are mostly "vertical" (white), and some that are mostly "horizontal" (black), along with some sites which are active switchers (purple). The numbered sites in Fig. 4 correspond to the time traces of $\sigma_{i}$ in Fig. 5. The local orientation at each site displays telegraph noise, but the nature of the telegraph noise is different for different sites. For example, $\sigma_{2}$ shows a few sharp spikes, whereas $\sigma_{6}$ and $\sigma_{7}$ show frequent switching. Notice that the noise is correlated: when $\sigma_{6}$ is "up," $\sigma_{7}$ is also "up"; however, when $\sigma_{6}$ is down, $\sigma_{7}$ becomes "active" and switches rapidly. This behavior indicates correlated switchers, which is the essence of the interacting model we use. One might imagine that such correlations could be detected via simultaneous measurements with two (or more) STM tips; however, that would require placing the tips closer together than can be achieved with current setups. We therefore focus on developing a criterion for detecting correlated noise with a single tip.

One way to characterize the temporal behavior of the local noise is to study the autocorrelation function, $\Psi_{i}(t)$ $=\frac{1}{t_{\max }-t} \sum_{t^{\prime}=1}^{t_{\max }-t} \sigma_{i}\left(t^{\prime}\right) \sigma_{i}\left(t^{\prime}+t\right)-\frac{1}{t_{\max }} \sum_{t=1}^{t_{\max }} \sigma_{i}(t)$. However, we have found that the autocorrelation is prone to large statistical errors at long times, and hence provides an unreliable characterization of the data. The origin of this issue lies in the interactions of the system. Because each site is interacting with several other sites, each site responds at several different time scales. The true asymptotic behavior of the local autocorrelation is not achieved until well beyond all of these time scales.

For this reason, we instead use the "on-the-fly" reblocking algorithm ${ }^{18}$ described below, which is efficient, robust, and lends itself easily to real-time signal processing during data collection. A graphical representation of this method is shown in Fig. 6. A block spin is defined to be the average spin at a particular site, averaged over $m$ consecutive timesteps, where $m$ is the block size. As the Monte Carlo simulation proceeds, we update the values of the most recent complete blocks of sizes that are powers of two: $m$ $=1,2,4,8,16, \ldots$. We also accumulate the sums and sums of squares of block spins so that we can ultimately construct the block averages and block variances. We use unbiased estimators for the variances so that for, e.g., a 16-element time series at block size $m=2$,

$$
\sigma_{(2)}=\frac{1}{8}\left[\frac{\sigma_{1}+\sigma_{2}}{2}+\cdots+\frac{\sigma_{15}+\sigma_{16}}{2}\right],
$$

$\left\langle\left(\Delta \sigma_{(2)}\right)^{2}\right\rangle=\frac{1}{7}\left[\left(\frac{\sigma_{1}+\sigma_{2}}{2}\right)^{2}+\cdots+\left(\frac{\sigma_{15}+\sigma_{16}}{2}\right)^{2}\right]-\frac{8}{7}\left[\sigma_{(2)}\right]^{2}$.

This procedure requires minimal computational effort, and furthermore requires the storage of only $O\left(\log _{2} N\right)$ numbers

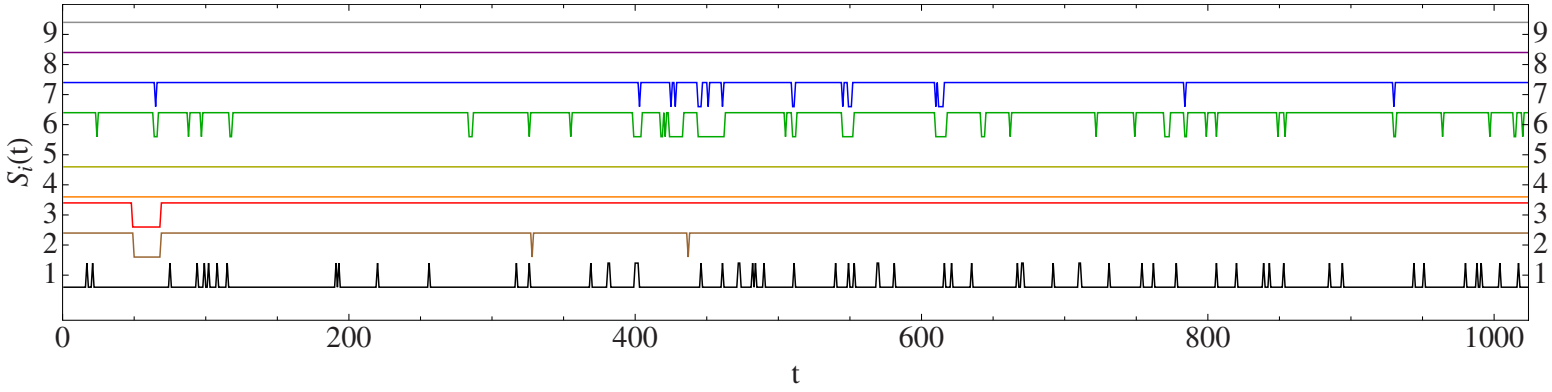

FIG. 5. (Color online) Time traces of the nine sites denoted in Fig. 4, with $R=2.5 \mathrm{~J}$ and $T=0.5 \mathrm{~J}$. . 


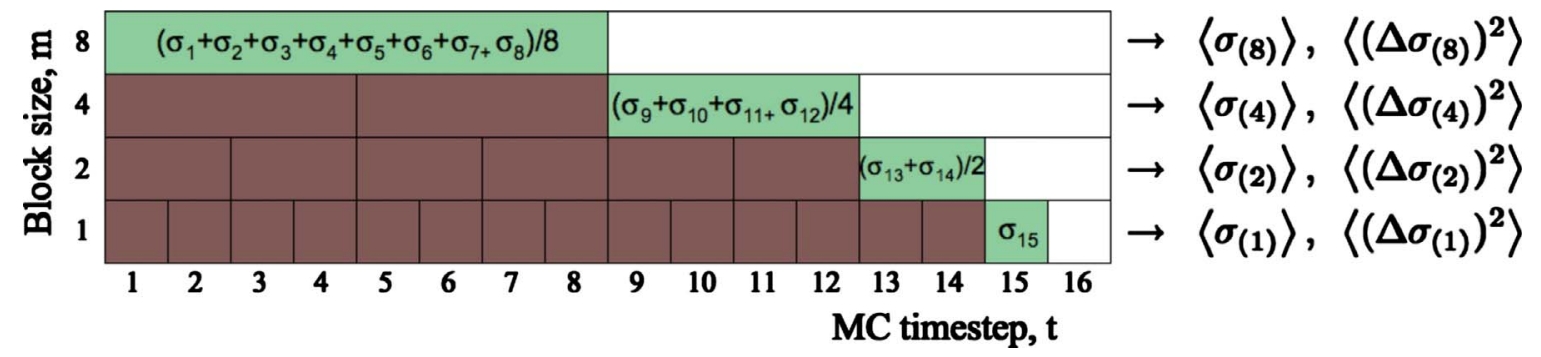

FIG. 6. (Color online) Visualization of the "real-time" reblocking algorithm for the spin history at a single site. The horizontal axis represents Monte Carlo time (or real time in a discrete time series from experiment); the vertical axis represents the block size, $m$. Dark areas represent previously computed blockspin values that have already have been discarded from memory. White areas are yet to be calculated. The lightly shaded (green) areas denote the current state which must be held in memory, of order $O\left(\log _{2} \mathrm{~N}\right)$ at time step $t=N$.

in memory, making the method amenable to real-time data analysis. The variance $\left\langle(\Delta \sigma)^{2}\right\rangle_{m}$ as a function of the temporal block size $m$ can then be used to distinguish a telegraph signal that is free of spatial correlations (i.e., one due to a stationary random telegraph signal) from a telegraph signal due to an interacting model, the spatial correlations of which contribute multiple time scales to each local switcher.

We first describe the results of this analysis for an uncorrelated, independent switcher [a stationary random telegraph signal (RTS)], and then contrast it with local telegraph noise from an interacting model, which encodes information about the correlations. At the block size $m$, the block variance can be expressed as

$$
\left\langle\left(\Delta \sigma_{(m)}\right)^{2}\right\rangle=\frac{1}{m^{2}}\left\langle\sigma_{(m)}^{2}\right\rangle-\frac{1}{m^{2}}\left\langle\sigma_{(m)}\right)^{2}=\frac{1}{m} \sum_{t=-m}^{m}(m-|t|) \psi(t),
$$

where the average $\langle\cdots\rangle$ is taken over all blocks of size $m$, and $\psi(t)$ is the time-dependent part of the autocorrelation function $\Psi(t)$. For a stationary discrete-time RTS, ${ }^{16}$ the autocorrelation is exponential (corresponding to a Lorentzian power spectrum),

$$
\psi(t)=a e^{-|t| / \tau}
$$

$$
\text { where } a=\frac{4 p q}{(p+q)^{2}}, \quad e^{-1 / \tau}=\lambda=1-p-q,
$$

where $p(q)$ is the conditional probability of switching from the "up" ("down") state to the "down" ("up") state at the next time step, and $\tau$ is the resulting characteristic time scale. Thus the block variance is

$$
\left\langle\left(\Delta \sigma_{(m)}\right)^{2}\right\rangle=a \frac{m-m \lambda^{2}+2 \lambda\left(\lambda^{m}-1\right)}{m^{2}(\lambda-1)^{2}} .
$$

Figure 7 shows the normalized block variance $\left\langle\left(\Delta \sigma_{(m)}\right)^{2}\right\rangle /\left\langle\left(\Delta \sigma_{(1)}\right)^{2}\right\rangle$ vs $m$ for uncorrelated switchers with various $\tau$. The small $m$ behavior is a horizontal line on a log-log plot, whereas the large $m$ behavior is a straight line corresponding to $\left\langle\left(\Delta \sigma_{(m)}\right)^{2}\right\rangle \propto 1 / m$. The extrapolated intersection of these two linear regimes may be used to extract a rough time scale $\tau_{i}$ for each site $i$,

$$
\tau_{i}=m_{\max }\left\langle\left(\Delta \sigma_{(m)}^{i}\right)^{2}\right\rangle /\left\langle\left(\Delta \sigma_{(1)}^{i}\right)^{2}\right\rangle,
$$

where $m_{\max }$ is the largest block size for which variance data is available. We will see that deviations from the horizontal line at low values of $m$ can be used as a local measure of spatial correlations.

In Fig. 8, we show the variance in our model, where spatial correlations have caused a deviation from the simple behavior of a stationary RTS. The curves correspond to the numbered sites in Fig. 4. A couple of these curves mimic that of a stationary RTS. For example, curve 3 begins with the horizontal line characteristic of a stationary RTS, and then crosses over to the $1 / \mathrm{m}$ behavior at a rather large time scale. In addition, curve 8 displays the $1 / \mathrm{m}$ behavior throughout the plot, consistent with a local stationary RTS with a rather short time scale. The rest of the curves in Fig. 8 show a striking deviation from the stationary RTS behavior, in that they begin at small $m$ with a slope which is in between a horizontal line and $1 / \mathrm{m}$ behavior. This may be taken as an indication of correlations. In several of the curves, multiple "knees" are evident within the same curve, further evidence of correlations, in that there are multiple time scales present in the behavior of a single site. This type of analysis is amenable to real-time data processing of a telegraph STM signal in an "active" region, in order to determine whether the noise is due to a local independent switcher (which may be due to,

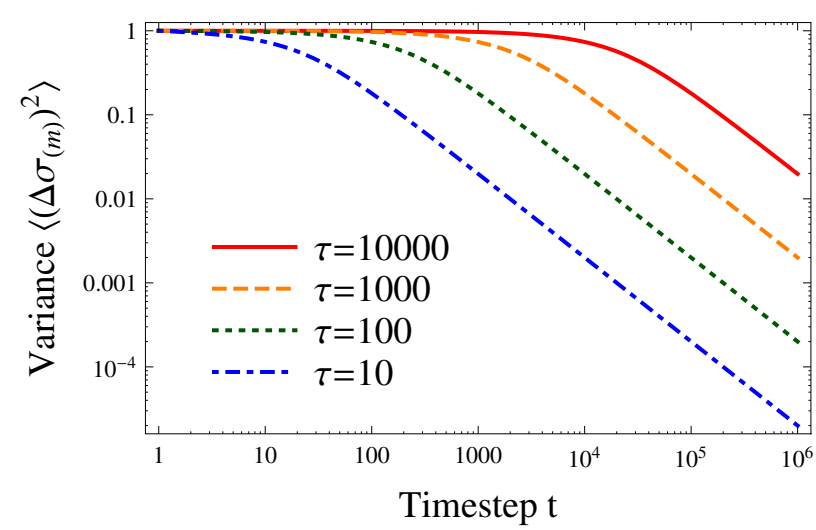

FIG. 7. (Color online) Normalized block variances $\left\langle\left(\Delta \sigma_{(m)}\right)^{2}\right\rangle /\left\langle\left(\Delta \sigma_{(1)}\right)^{2}\right\rangle$ vs block size $m$ of an uncorrelated switcher (stationary RTS) for various characteristic time scales $\tau$, showing the crossover from constant to $1 / \mathrm{m}$ behavior. 


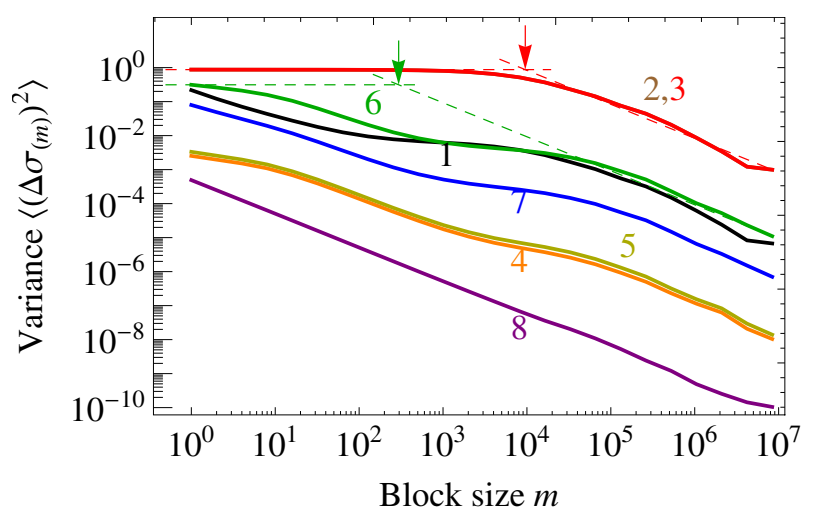

FIG. 8. (Color online) Block variances $V_{i}^{(m)}$ vs block size $m$ for the numbered sites in Fig. 4. For curves 3 and 6, the intersection of the dotted lines shows the characteristic time scale of those sites, $\tau_{i}$, as described in the text. While the results for curves 3 and 9 mimic that of a stationary RTS with a long and short time scale, respectively, the other timetraces show strong deviation from this behavior, a clear signature of the correlations in the system. Curve 9 is not shown because the corresponding spins have zero variance over the sampled time interval.

e.g., a "dirt" effect), or whether it is due to the more interesting case of correlations due to interactions among the electronic degrees of freedom. ${ }^{19}$ In addition, it is free from the windowing errors that an FFT would entail.

In the right column of Fig. 3, we plot maps of the local switching time scale, $\tau$, estimated from Eq. (8). These may be viewed as a map of the dynamic clusters, i.e., those sites which fluctuate together. Note that this is different from the identification of the low temperature domains. The behavior of the largest time scales as temperature is lowered (i.e., in the active regions) is consistent with an Arrhenius law. However, a single time scale is insufficient to characterize the local dynamics because of the correlations, and there are longer time scales in the active regions than can be properly represented with a $2 \mathrm{D}$ color plot.

\section{DISCUSSION}

We have argued that there should be telegraph noise in STM experiments on locally striped materials at intermediate temperatures. Our proposal can be summarized as follows: Using STM in, e.g., a tunneling asymmetry (TA) mode, the low temperature spatial map can be used to identify the low temperature domain structure. Then, place the tip on a domain boundary, preferably where several small domains are clustered, and take time traces of the signal at a few sites. If no telegraph noise is evident, raise the temperature, and repeat. At high enough temperature, switching of the stripe orientation should become thermally excited, resulting in local telegraph noise. We have furthermore proposed a criterion by which such noise can be analyzed in the time domain, in order to determine whether it is arising from a local, independent switcher, or whether it is instead arising from correlated fluctuations in an interacting model.
Important issues include which signal should be tracked, at what time scale do we expect telegraph noise to be evident, and what are good candidate materials for testing our proposal. Some evidence of stripe structure has been reported in STM on BSCCO (Refs. 20 and 21) in the 8-15 meV range, derived from measurements of $d I / d V$. However, spatial modulations in the low-energy response have also been attributed to quasiparticle interference. ${ }^{22,23}$ More dramatic evidence of locally unidirectional electronic domains, with minimal signal processing involved, has been reported in Dy-BSCCO and NCCOC (Ref. 3) via TA maps. Specifically, the ratio of the tunneling current at $\pm 150 \mathrm{mV}$ resulted in high contrast images of domains with local Ising symmetry, consistent with the model studied here. For the purposes of studying noise associated with these Ising domains, a time trace of the current near $\pm 150 \mathrm{mV}$ would be a good place to start. (Note that the magnitude of variation in signal in those experiments is on the order of $30 \%$.) The variance test proposed here, which depends on the time domain properties of a discrete signal change, would apply whether the "tip height" $z$ was held constant and a time trace of the current $I$ measured, or whether the current $I$ was held fixed and a time trace of $z$ was recorded.

It is worth emphasizing that telegraph noise has already been reported in the transport properties of a YBCO nanowire. ${ }^{5}$ In addition, similar behavior was noted in a BSCCO nanowire, ${ }^{24}$ although sufficient data was not gathered to demonstrate telegraph noise in a time trace. In this case, switches in the resistivity were observed starting above $T_{c}$ (since the resistivity is zero below $T_{c}$ ), and going as high as $T=150 \mathrm{~K}$. The noise was smaller in magnitude, and less frequent than the corresponding noise in YBCO. However, BSCCO is much more amenable to an STM experiment, and given that locally unidirectional domains have been observed in TA maps in Dy-BSCCO, this system is a good candidate for observing thermally excited stripe switching noise via STM.

In this paper, we argue that fluctuations of stripe orientations will produce correlated telegraph noise in scanning tunneling experiments, and we propose a method for analyzing such noise in the time domain. This does not, of course, rule out the possibility of correlated noise emerging from other sources (e.g., side-to-side stripe fluctuations, or fluctuations of other inhomogeneous patterns, such as checkerboards or quasiparticle interference patterns).

\section{CONCLUSIONS}

In conclusion, we have analyzed the local noise characteristics of the random field Ising model in the disordered phase. For materials with local stripe correlations in the presence of disorder, this model predicts that there will be thermally excited fluctuations in the stripe orientation. We propose that such fluctuations should result in correlated telegraph noise in the STM response near stripe domain boundaries as temperature is raised. The use of this method in conjunction with TA scans can establish whether there is a connection between the observed low- $T$ unidirectional, glassy domains in Na-CCOC and Dy-Bi2212, and the trans- 
port noise observed in YBCO and LSCO, and also put constraints on our model. We have furthermore proposed that the block variance of telegraph noise can be used as a purely local indicator of spatial correlations. The criterion can be applied to any local probe (AFM, MFM, etc.) and because it is accumulated directly in the time domain, it is amenable to real-time signal processing.

Note added in proof. Recent results on BSCCO confirm that an Ising nematic order parameter presents itself in much of the STM signal. ${ }^{27}$ The large cluster size is consistent with $\mathrm{BSCCO}$ being in the intermediate disorder regime of the model discussed in the present paper, in which disorder is weak within a plane, but strong between planes. ${ }^{28}$

\section{ACKNOWLEDGMENTS}

It is a pleasure to thank G. Aeppli, L. Biedermann, J. C. Davis, E. Fradkin, T. Hanaguri, E. Hudson, S. Kivelson, B. Phillabaum, and M. Weissman for helpful discussions. This work was supported by Research Corporation and by NSF Grants No. DMR 08-04748 and No. DMR 03-25939 ITR (MCC).
${ }^{1}$ E. W. Carlson, K. A. Dahmen, E. Fradkin, and S. A. Kivelson, Phys. Rev. Lett. 96, 097003 (2006).

${ }^{2}$ J. M. Tranquada, arXiv:cond-mat/0512115 (unpublished).

${ }^{3}$ Y. Kohsaka, C. Taylor, K. Fujita, A. Schmidt, C. Lupien, T. Hanaguri, M. Azuma, M. Takano, H. Eisaki, H. Tagaki, S. Uchida, and J. C. Davis, Science 315, 1380 (2007).

${ }^{4}$ S. A. Kivelson, I. P. Bindloss, E. Fradkin, V. Oganesyan, J. M. Tranquada, A. Kapitulnik, and C. Howald, Rev. Mod. Phys. 75, 1201 (2003).

${ }^{5}$ J. A. Bonetti, D. S. Caplan, D. J. Van Harlingen, and M. B. Weissman, Phys. Rev. Lett. 93, 087002 (2004).

${ }^{6}$ I. Raičević, J. Jaroszyński, D. Popović, C. Panagopoulos, and T. Sasagawa, Phys. Rev. Lett. 101, 177004 (2008).

${ }^{7}$ L. Fruchter, H. Raffy, and Z. Z. Li, Phys. Rev. B 76, 212503 (2007).

${ }^{8}$ A. Del Maestro, B. Rosenow, and S. Sachdev, Phys. Rev. B 74, 024520 (2006).

${ }^{9}$ J. A. Robertson, S. A. Kivelson, E. Fradkin, A. C. Fang, and A. Kapitulnik, Phys. Rev. B 74, 134507 (2006).

${ }^{10}$ Other examples of an external perturbation which breaks the rotational symmetry of the lattice and would therefore tend to align stripes include strain, high currents (Ref. 25) and uniaxial pressure.

${ }^{11}$ S. Kartha, T. Castán, J. A. Krumhansl, and J. P. Sethna, Phys. Rev. Lett. 67, 3630 (1991).

${ }^{12}$ A. J. Bray and M. A. Moore, J. Phys. C 18, L927 (1985).

${ }^{13}$ In some magnetic systems Kawasaki dynamics may be more appropriate, as they conserve local magnetization. However, in our problem there is no reason for nematicity to be locally conserved, so Glauber dynamics are appropriate. Furthermore, because the switching of the local nematic orientation (a single Ising variable in our coarse-grained model) involves many electrons, we do not expect this to be a coherent quantum process. Therefore, we treat the dynamics classically, rather than using a Caldeira-Leggett model.
${ }^{14}$ Recall that at $100 \mathrm{~K}$, telegraph noise in the YBCO nanowire has a timescale of $10-50 \mathrm{~s}$. By $4.2 \mathrm{~K}$, thermal fluctuations in stripe orientation should be frozen out in that material. Likewise, fluctuations in stripe orientation should be similarly frozen out in $\mathrm{BSCCO}$ and $\mathrm{NCCOC}$ at $4.2 \mathrm{~K}$.

${ }^{15}$ M. B. Weissman, Rev. Mod. Phys. 60, 537 (1988).

${ }^{16}$ S. Machlup, J. Appl. Phys. 25, 341 (1954).

${ }^{17}$ Second spectra, another frequency-domain criterion, may also be used (Ref. 15).

${ }^{18}$ D. R. Kent IV, R. P. Muller, A. G. Anderson, W. A. Goddard III, and M. T. Feldmann, J. Comput. Chem. 28, 2309 (2007).

${ }^{19}$ Note that we have not focused on critical behavior or on scaling behavior (Refs. 12 and 26). Comparing those type of predictions to STM requires that the measurements be averaged over a large field of view. One advantage of our proposal is the focus on local physics, without the need for spatial averages.

${ }^{20}$ C. Howald, H. Eisaki, N. Kaneko, and A. Kapitulnik, Proc. Natl. Acad. Sci. U.S.A. 100, 9705 (2003).

${ }^{21}$ A. Fang, C. Howald, N. Kaneko, M. Greven, and A. Kapitulnik, Phys. Rev. B 70, 214514 (2004).

${ }^{22}$ T. Hanaguri, Y. Kohsaka, J. C. Davis, C. Lupien, I. Yamada, M. Azuma, M. Takano, K. Ohishi, M. Ono, and H. Takagi, Nat. Phys. 3, 865 (2007).

${ }^{23}$ J. E. Hoffman, K. McElroy, D.-H. Lee, K. M. Lang, H. Eisaki, S. Uchida, and J. C. Davis, Science 297, 1148 (2002).

${ }^{24}$ D. Van Harlingen (private communication).

${ }^{25}$ C. Reichhardt, C. J. Olson Reichhardt, and A. R. Bishop, EPL 72, 444 (2005).

${ }^{26}$ D. S. Fisher, Phys. Rev. Lett. 56, 416 (1986).

${ }^{27}$ M. J. Lawler, K. Fujita, J. Lee, A. R. Schmidt, Y. Kohsaka, C. K. Kim, H. Eisaki, S. Uchida, J. C. Davis, J. P. Sethna, and E.-A. Kim, Nature (London) (to be published).

${ }^{28}$ O. Zachar and I. Zaliznyak, Phys. Rev. Lett. 91, 036401 (2003). 